\begin{tabular}{|c|l|}
\hline Title & Inequalities associated with dilations \\
\hline Author(s) & Ozawa, Tohru; Sasaki, Hironobu \\
\hline Citation & Hokkaido University Preprint Series in Mathematics, 861, 1-12 \\
\hline Issue Date & 2007 \\
\hline DOI & 10.14943/84011 \\
\hline Doc URL & http://hdl.handle.net/2115/69670 \\
\hline Type & bulletin (article) \\
\hline File Information & pre861.pdf \\
\hline
\end{tabular}

Instructions for use 


\title{
INEQUALITIES ASSOCIATED WITH DILATIONS
}

\author{
TOHRU OZAWA AND HIRONOBU SASAKI*
}

\begin{abstract}
Some properties of distributions $f$ satisfying $x \cdot \nabla f \in L^{p}\left(\mathbb{R}^{n}\right), 1 \leq p<\infty$, are studied. The operator $x \cdot \nabla$ is the generator of a semi-group of dilations. We first give Sobolev type inequalities with respect to the operator $x \cdot \nabla$. Using the inequalities, we also show that if $f \in L_{\text {loc }}^{p}\left(\mathbb{R}^{n}\right), x \cdot \nabla f \in L^{p}\left(\mathbb{R}^{n}\right)$ and $|x|^{n / p}|f(x)|$ vanishes at infinity, then $f$ belongs to $L^{p}\left(\mathbb{R}^{n}\right)$. One of the Sobolev type inequalities is shown to be equivalent to the Hardy inequality in $L^{2}\left(\mathbb{R}^{n}\right)$.
\end{abstract}

\section{INTRODUCTION}

In this paper, we study some properties of distributions $f \in \mathcal{D}^{\prime}(\Omega)$ satisfying $x \cdot \nabla f \in$ $L^{p}(\Omega)$. Here, $\Omega \subset \mathbb{R}^{n}$ is an open set, $\mathcal{D}^{\prime}(\Omega)$ is the set of all distributions on $\Omega, x \cdot \nabla=$ $\sum_{j=1}^{n} x_{j} \partial_{j}, x=\left(x_{1}, \cdots, x_{n}\right) \in \Omega$ and $\partial_{j} f$ is a weak derivative of $f$ with respect to $x_{j}$. The operator $x \cdot \nabla$ is well-known as the generator of a semi-group of dilations $\{T(t)\}_{t \geq 0}$ defined by

$$
(T(t) g)(x)=g\left(e^{t} x\right), \quad g: \mathbb{R}^{n} \rightarrow \mathbb{C}, \quad x \in \mathbb{R}^{n} .
$$

Let us recall the Sobolev inequality. For a Banach space $A$, we denote the norm of $A$ by $\|\cdot \mid A\|$. It is well-known that if $1<p, p^{*}<\infty$ and

$$
\frac{1}{p}=\frac{1}{p^{*}}-\frac{1}{n}
$$

then we have the Sobolev inequality:

$$
\left\|g\left|L^{p}\left(\mathbb{R}^{n}\right)\|\leq C(p)\| \nabla g\right| L^{p^{*}}\left(\mathbb{R}^{n}\right)\right\| .
$$

Remark that the constant $C(p)$ in (1.2) is independent of $g$. For any $\lambda>0$, we obtain

$$
\lambda^{-n / p}\left\|h\left|L^{p}\left(\mathbb{R}^{n}\right)\left\|\leq \lambda^{-n / p^{*}+1} C(p)\right\| \nabla h\right| L^{p^{*}}\left(\mathbb{R}^{n}\right)\right\|
$$

by substituting $g(x)=h(\lambda x)$ into (1.2). Therefore, we observe that (1.1) is a necessary condition.

2000 Mathematics Subject Classification. 26D10, 46E35.

Key words and phrases. Inequalities; Generator of semi-group of dilations; Sobolev's inequality; Poincaré's inequality; Hardy's inequality.

* Supported by Research Fellowships of the Japan Society for the Promotion of Science for Young Scientists. 
Throughout this paper, we consider the following Sobolev type inequality with respect to the operator $x \cdot \nabla$ instead of $\nabla$ :

$$
\left\|g\left|L^{p}\left(\mathbb{R}^{n}\right)\left\|\leq C^{\prime}(p)\right\| x \cdot \nabla g\right| L^{q}\left(\mathbb{R}^{n}\right)\right\| .
$$

Substituting $g(x)=h(\lambda x)$ into (1.3), we observe that $p=q$ is a necessary condition to obtain (1.3). Later, we shall prove that (1.3) holds if $1 \leq p=q<\infty, f \in L^{p}$ and $x \cdot \nabla f \in L^{p}$.

To state our results, we list some notation which will be used later. For $1 \leq p \leq \infty$, we put $L^{p}=L^{p}\left(\mathbb{R}^{n}\right)$ and $\|\cdot\|_{p}=\left\|\cdot \mid L^{p}\right\|$. For $k=0,1, \cdots$ and for an open set $\Omega \subset \mathbb{R}^{n}$, We denote by $C_{c}^{\infty}(\Omega)$ the set of all $C^{\infty}$-functions with compact support in $\Omega$. For $1 \leq p \leq \infty$ and for an open set $\Omega \subset \mathbb{R}^{n}$, let $W_{0}^{1, p}(\Omega)$ be the completion of $C_{c}^{\infty}(\Omega)$ with respect to

$$
\left\|g\left|W^{1, p}(\Omega)\|=\| g\right| L^{p}(\Omega)\right\|+\left\|\nabla g \mid L^{p}(\Omega)\right\| .
$$

Let $\zeta: \mathbb{R} \rightarrow \mathbb{R}$ be an even, $C^{\infty}$-function satisfying

- $0 \leq \zeta \leq 1$,

- $\zeta(r)=1$ if $|r| \leq 1$,

- $\zeta(r)=0$ if $|r| \geq 2$.

We are ready to state our first result.

Theorem 1.1. Let $n \geq 1$.

(i) Assume that $\Omega \subset \mathbb{R}^{n}$ is an open set. If $1 \leq p<\infty, f \in W_{0}^{1, p}(\Omega)$ and $x \cdot \nabla f \in L^{p}(\Omega)$, then we have

$$
\left\|f\left|L^{p}(\Omega)\left\|\leq \frac{p}{n}\right\| x \cdot \nabla f\right| L^{p}(\Omega)\right\|
$$

(ii) If $1 \leq p<\infty, f \in L^{p}$ and $x \cdot \nabla f \in L^{p}$, then (1.4) holds. That is, we have

$$
\|f\|_{p} \leq \frac{p}{n}\|x \cdot \nabla f\|_{p}
$$

(iii) If $f \in C^{1}\left(\mathbb{R}^{n}\right)$ and if there exist positive numbers $\varepsilon$ and $R$ such that

$$
\operatorname{supp} f \subset\left\{x \in \mathbb{R}^{n} ; \varepsilon \leq|x| \leq R\right\},
$$

then we have

$$
\|f\|_{\infty} \leq \ln \left(\frac{R}{\varepsilon}\right)\|x \cdot \nabla f\|_{\infty} .
$$

The proof of Theorem 1.1 will be given in Section 2. We now list three remarks on Theorem 1.1.

Remark 1. A constant function $f_{C}(x):=C \neq 0$ is a typical example of a function which dose not satisfy (1.4). Remark that

$f_{C} \in W^{1, p}(\Omega) \backslash W_{0}^{1, p}(\Omega)$ if $1 \leq p<\infty$ and $\Omega$ has a finite measure, $f_{C} \in L_{l o c}^{p} \backslash L^{p} \quad$ if $1 \leq p<\infty$. 
Remark 2. The condition (1.6) is a necessary condition in some sense. For $0<\delta<1 / 2$ and $0<\varepsilon<R<\infty$, we define functions $f_{\delta, R}^{1}, f_{\delta, \varepsilon}^{2}$ and $f_{\delta}^{3}$ by

$$
\begin{aligned}
& f_{\delta, R}^{1}(x)= \begin{cases}(\ln \delta)^{-1} \zeta\left(\frac{|x|}{R}\right) \ln \sqrt{\frac{|x|^{2}}{R^{2}}+\delta^{2}} & \text { if } 0 \leq|x| \leq R, \\
0 & \text { otherwise, }\end{cases} \\
& f_{\delta, \varepsilon}^{2}(x)= \begin{cases}0 & \text { if } 0 \leq|x| \leq \varepsilon, \\
(\ln \delta)^{-1} \zeta\left(\frac{\varepsilon}{|x|}\right)\left\langle\frac{x}{\varepsilon}\right\rangle^{-\delta} \ln \sqrt{\frac{\varepsilon^{2}}{|x|^{2}}+\delta^{2}} & \text { otherwise, }\end{cases} \\
& f_{\delta}^{3}(x)=\langle x\rangle^{-\delta},
\end{aligned}
$$

respectively. Here, $\langle x\rangle=\left(1+|x|^{2}\right)^{1 / 2}$. Then we see that $f_{\delta, R}^{1}, f_{\delta, \varepsilon}^{2}$ and $f_{\delta}^{3}$ are $C^{\infty}$-functions vanishing at infinity and satisfy

$$
\begin{aligned}
\operatorname{supp} f_{\delta, R}^{1} & =\left\{x \in \mathbb{R}^{n} ; 0 \leq|x| \leq R\right\}, \\
\operatorname{supp} f_{\delta, \varepsilon}^{2} & =\left\{x \in \mathbb{R}^{n} ;|x| \geq \varepsilon\right\}, \\
\operatorname{supp} f_{\delta}^{3} & =\mathbb{R}^{n} .
\end{aligned}
$$

Furthermore, we observe that

$$
\lim _{\delta \rightarrow 0+} \frac{\left\|g_{\delta}\right\|_{\infty}}{\left\|x \cdot \nabla g_{\delta}\right\|_{\infty}}=\infty, \quad g_{\delta}=f_{\delta, R}^{1}, f_{\delta, \varepsilon}^{2}, f_{\delta}^{3}
$$

Remark 3. The number $p / n$ appearing in (1.5) is the best constant. In fact, if we take

$$
f_{\varepsilon}(x)= \begin{cases}|x|^{-n / p+\varepsilon} & \text { for }|x|<1 \\ |x|^{-n / p-\varepsilon} & \text { for }|x| \geq 1\end{cases}
$$

then we have

$$
x \cdot \nabla f_{\varepsilon}(x)= \begin{cases}\left(-\frac{n}{p}+\varepsilon\right)|x|^{-n / p+\varepsilon} & \text { if }|x|<1, \\ \left(-\frac{n}{p}-\varepsilon\right)|x|^{-n / p-\varepsilon} & \text { if }|x|>1\end{cases}
$$

in the $L^{p}$-sense. Hence we see that

$$
\frac{\left\|f_{\varepsilon}\right\|_{p}}{\left\|x \cdot \nabla f_{\varepsilon}\right\|_{p}} \geq 2^{\frac{1}{p}}\left\{\left(\frac{n}{p}-\varepsilon\right)^{p}+\left(\frac{n}{p}+\varepsilon\right)^{p}\right\}^{-\frac{1}{p}} \rightarrow \frac{p}{n}
$$

as $\varepsilon \rightarrow 0$. Moreover, if we take $g(x)=e^{-|x|^{2}}$, then we have

$$
\frac{\|g\|_{1}}{\|x \cdot \nabla g\|_{1}}=\frac{1}{n} \text {. }
$$

Remark 4. If $\Omega \subset \mathbb{R}^{n}$ is bounded, then (1.4) implies the usual Poincaré inequality. See $[5,8]$ for related results. 
As the application of the inequality (1.5), we give the following three propositions: The first proposition is concerned with a function space $\mathfrak{L}^{p}:=\left\{f \in L^{p} ; x \cdot \nabla f \in L^{p}\right\}$ equipped with a semi-norm

$$
\left\|f \mid \mathfrak{L}^{p}\right\|:=\|x \cdot \nabla f\|_{p}
$$

We shal prove that $\mathfrak{L}^{p}$ becomes a Banach space. Proposition 1.3, which is the second application, indicates that if a function $f \in L_{\mathrm{loc}}^{p}$ with $x \cdot \nabla f \in L^{p}$ satisfies some decreasing condition, then $f$ belongs to $L^{p}$. The third application Proposition 1.4 means that if $n \geq 3$ and $p=2$, then (1.5) is equivalent to the Hardy inequality.

Proposition 1.2. Let $n \geq 1$ and $1 \leq p<\infty$. Then the semi-normed space $\left(\mathfrak{L}^{p},\left\|\cdot \mid \mathfrak{L}^{p}\right\|\right)$ becomes a Banach space. Furthermore, the embedding operator $\iota: \mathfrak{L}^{p} \hookrightarrow L^{p}$ has the norm

$$
\left\|\iota \mid \mathfrak{L}^{p} \rightarrow L^{p}\right\|=\frac{p}{n} .
$$

Proof. By (1.5), we find that $f=0$ if and only if $\left\|f \mid \mathfrak{L}^{p}\right\|=0$. Thus, $\left\|\cdot \mid \mathfrak{L}^{p}\right\|$ is a norm of $\mathfrak{L}^{p}$. Let $\left\{f_{m}\right\}_{m=1}^{\infty}$ be a Cauchy sequence in $\mathfrak{L}^{p}$. From (1.5), there exist $f \in L^{p}$ and $g \in L^{p}$ such that

$$
\lim _{m \rightarrow \infty} f_{m}=f, \quad \lim _{m \rightarrow \infty} x \cdot \nabla f_{m}=g
$$

in $L^{p}$. For any $\varphi \in C_{c}^{\infty}\left(\mathbb{R}^{n}\right)$, it follows that

$$
\begin{aligned}
\int_{\mathbb{R}^{n}}(x \cdot \nabla f) \varphi d x & =-\int_{\mathbb{R}^{n}} f \operatorname{div}(x \varphi) d x \\
& =-\lim _{m \rightarrow \infty} \int_{\mathbb{R}^{n}} f_{m} \operatorname{div}(x \varphi) d x \\
& =\lim _{m \rightarrow \infty} \int_{\mathbb{R}^{n}}\left(x \cdot \nabla f_{m}\right) \varphi d x \\
& =\int_{\mathbb{R}^{n}} g \varphi d x .
\end{aligned}
$$

Therefore, we have $x \cdot \nabla f=g \in L^{p}$ and $\mathfrak{L}^{p}$ is complete. By Remark 3, (1.9) holds.

In Theorem 1.1, one of the main assumptions is that $f \in L^{p}$. As we see in Remark 1 above, the last space is not generalized to $L_{l o c}^{p}$. The following proposition shows a sufficient condition for the condition that $f \in L^{p}$.

Proposition 1.3. Let $n \geq 1$ and $1 \leq p<\infty$. Assume that $f \in \mathcal{D}^{\prime}\left(\mathbb{R}^{n}\right)$ is measurable on $\mathbb{R}^{n}$ and that $x \cdot \nabla f \in L^{p}$. If there exists $\left\{\phi_{l}\right\}_{l=1}^{\infty} \subset C_{c}^{\infty}\left(\mathbb{R}^{n}\right)$ such that

(i) $\sup _{l>1}\left\|\phi_{l}\right\|_{\infty}<\infty$,

(ii) $\lim _{l \rightarrow \infty} \phi_{l}(x)=1$ for a.e. $x$,

(iii) $\phi_{l} f \in L^{p}$, 
(iv) $\liminf \inf _{l \rightarrow \infty}\left\|\left(x \cdot \nabla \phi_{l}\right) f\right\|_{p}=0$

then $f \in L^{p}$.

Remark 5. In Section 3 below, we show some corollaries of the above proposition. In particular, we shall show that if $f \in L_{\mathrm{loc}}^{p}$ satisfies $x \cdot \nabla f \in L^{p}$ and if $|x|^{n / p}|f(x)|$ vanishes at infinity, then $f$ belongs to $L^{p}$.

Proof of Proposition 1.3. By (iii) and (1.5), we have

$$
\left\|\phi_{l} f\right\|_{p} \leq \frac{p}{n}\left\{\left\|\left(x \cdot \nabla \phi_{l}\right) f\right\|_{p}+\left\|\phi_{l}(x \cdot \nabla f)\right\|_{p}\right\}
$$

for any $l \geq 1$. Since $x \cdot \nabla f \in L^{p}$, we see from (i), (ii) and the Lebesgue dominated theorem that

$$
\lim _{l \rightarrow \infty}\left\|\phi_{l}(x \cdot \nabla f)\right\|_{p}=\|x \cdot \nabla f\|_{p}
$$

By (iv), we hence obtain

$$
\liminf _{l \rightarrow \infty}\left\|\phi_{l} f\right\|_{p} \leq \frac{p}{n}\|x \cdot \nabla f\|_{p}
$$

It follows from (ii) and the Fatou lemma that

$$
\begin{aligned}
\|f\|_{p}^{p} & =\int_{\mathbb{R}^{n}} \liminf _{l \rightarrow \infty}\left|\left(\phi_{l} f\right)(x)\right|^{p} d x \\
& \leq \liminf _{l \rightarrow \infty} \int_{\mathbb{R}^{n}}\left|\left(\phi_{l} f\right)(x)\right|^{p} d x \\
& =\liminf _{l \rightarrow \infty}\left\|\phi_{l} f\right\|_{p}^{p} .
\end{aligned}
$$

Thus, we see that $f \in L^{p}$.

The inequality (1.5) is equivalent to Hardy's inequality if $p=2$. To be more specific, we have:

Proposition 1.4. Let $n \geq 3$. Then the following two statements are equivalent:

(a) For any $f \in C_{c}^{\infty}\left(\mathbb{R}^{n} \backslash\{0\}\right)$, we have

$$
\|f\|_{2} \leq \frac{2}{n}\|x \cdot \nabla f\|_{2} .
$$

(b) For any $g \in C_{c}^{\infty}\left(\mathbb{R}^{n} \backslash\{0\}\right)$, we have

$$
\left\|\frac{g}{|x|}\right\|_{2} \leq \frac{2}{n-2}\left\|\frac{x}{|x|} \cdot \nabla g\right\|_{2} .
$$


Remark 6. The original Hardy inequality was given by [6]. Later, a lot of generalized Hardy inequalities are studied (see, e.g., $[1,2,3,4,7,9]$ ). In particular, it was shown that

$$
\left\|\frac{g}{|x|}\right\|_{p} \leq \frac{p}{n-p}\|\nabla g\|_{p}, \quad g \in W^{1, p}\left(\mathbb{R}^{n}\right), \quad p<n .
$$

The constant $p /(n-p)$ is optimal. Since $C_{c}^{\infty}\left(\mathbb{R}^{n} \backslash\{0\}\right)$ is dense in $W^{1,2}\left(\mathbb{R}^{n}\right)$ for $n \geq 3$, by using Theorem 1.1,(ii), the above proposition and (1.12), we can see that (1.11) holds for any $W^{1,2}\left(\mathbb{R}^{n}\right)$. If we put $g_{\varepsilon}=|x| f_{\varepsilon}$, where $f_{\varepsilon}$ have been given in Remark 3 , then we have

$$
\frac{\left\|g_{\varepsilon} /|x|\right\|_{2}}{\left\|(x /|x|) \cdot \nabla g_{\varepsilon}\right\|_{2}} \geq 2^{\frac{1}{2}}\left\{\left(\frac{n}{2}-1-\varepsilon\right)^{2}+\left(\frac{n}{2}-1+\varepsilon\right)^{2}\right\}^{-\frac{1}{2}} \rightarrow \frac{2}{n-2}
$$

as $\varepsilon \rightarrow 0$. Therefore, the constant $2 /(n-2)$ is optimal.

Proof of Proposition 1.4. We first prove that the statement (a) implies (b). Put $g=|x| f$. Then we obtain

$$
\|x \cdot \nabla f\|_{2}=\left\|-\frac{g}{|x|}+\frac{x}{|x|} \cdot \nabla g\right\|_{2}^{2}=\left\|\frac{g}{|x|}\right\|_{2}^{2}-2 \Re\left\langle\frac{g}{|x|}, \frac{x}{|x|} \cdot \nabla g\right\rangle+\left\|\frac{x}{|x|} \cdot \nabla g\right\|_{2}^{2},
$$

where $\langle\cdot, \cdot\rangle$ denotes the inner product in $L^{2}$. Since

$$
-2 \Re\left\langle\frac{g}{|x|}, \frac{x}{|x|} \cdot \nabla g\right\rangle=-\left\langle\frac{x}{|x|^{2}}, \nabla|g|^{2}\right\rangle=\left\langle\operatorname{div}\left(\frac{x}{|x|^{2}}\right),|g|^{2}\right\rangle=\left\langle\frac{n-2}{|x|^{2}},|g|^{2}\right\rangle,
$$

It follows from the statement (a) and (1.13) that

$$
\left\|\frac{g}{|x|}\right\|_{2}^{2} \leq \frac{4}{n^{2}}\left((n-1)\left\|\frac{g}{|x|}\right\|_{2}^{2}+\left\|\frac{x}{|x|} \cdot \nabla g\right\|_{2}^{2}\right)
$$

which implies (1.11).

Conversely, we assume that (b) holds. We put $f=g /|x|$. Then we have

$$
\left\|\frac{x}{|x|} \cdot \nabla(|x| f)\right\|_{2}^{2}=\|f+x \cdot \nabla f\|_{2}^{2}=\|f\|_{2}^{2}+2 \Re\langle x f, \nabla f\rangle+\|x \cdot \nabla f\|_{2}^{2}
$$

Since $2 \Re\langle x f, \nabla f\rangle=-n\|f\|_{2}^{2}$, we see from the statement (b) that

$$
\|f\|_{2}^{2} \leq \frac{4}{(n-2)^{2}}\left(\|x \cdot \nabla f\|_{2}^{2}-(n-1)\|f\|_{2}^{2}\right),
$$

which implies (1.10). 


\section{Proof of Theorem 1.1}

In this section, we give a proof of Theorem 1.1. For this purpose, we prepare some notation. For $\lambda>0$ and for $g: \mathbb{R}^{n} \rightarrow \mathbb{C},\left(\delta_{\lambda} g\right)(x)=g(\lambda x)$. For $\lambda>0$ and for $\Omega \subset \mathbb{R}^{n}$, $\lambda \Omega=\{\lambda \omega ; \omega \in \Omega\}$. Let $\varphi \in C_{c}^{\infty}\left(\mathbb{R}^{n}\right)$ be a positive function such that $\int \varphi=1$ and $\operatorname{supp} \varphi \subset\left\{x \in \mathbb{R}^{n} ;|x| \leq 1\right\}$. For $\lambda>0$, we set $\zeta_{\lambda}(x)=\zeta(\lambda|x|)$ and $\varphi_{\lambda}=\lambda^{-n} \delta_{\lambda^{-1}} \varphi$.

Proposition 2.1. Let $n \geq 1$ and $1 \leq p<\infty$. Assume that $\Omega \subset \mathbb{R}^{n}$ is an open set. If $f \in C_{c}^{\infty}(\Omega)$, then we have (1.4).

Proof. For $\phi: \Omega \rightarrow \mathbb{C}$, we put

$$
\widetilde{\phi}(x)=\left\{\begin{array}{cl}
\phi(x) & \text { if } x \in \Omega \\
0 & \text { otherwise. }
\end{array}\right.
$$

Then we have

$$
\begin{aligned}
\left\|\delta_{\lambda} \widetilde{\phi} \mid L^{p}(\Omega)\right\|^{p} & =\int_{\Omega}|\widetilde{\phi}(\lambda x)|^{p} d x \\
& =\lambda^{-n} \int_{\lambda \Omega}|\widetilde{\phi}(x)|^{p} d x \\
& \leq \lambda^{-n} \int_{\Omega}|\widetilde{\phi}(x)|^{p} d x \\
& =\lambda^{-n}\left\|\phi \mid L^{p}(\Omega)\right\|^{p} .
\end{aligned}
$$

For any $\lambda>0$ and $x \in \Omega$,

$$
\begin{aligned}
\left(\delta_{\lambda} \widetilde{f}-\widetilde{f}\right)(x) & =\widetilde{f}(\lambda x)-\widetilde{f}(x) \\
& =\int_{1}^{\lambda} \frac{\partial}{\partial \alpha} \widetilde{f}(\alpha x) d \alpha \\
& =\int_{1}^{\lambda} \alpha^{-1}\left(\delta_{\alpha} \widetilde{x \cdot \nabla f}\right)(x) d \alpha
\end{aligned}
$$

For any $\lambda>1$, we see from (2.1) and (2.2) that

$$
\begin{aligned}
\left(1-\lambda^{-n / p}\right)\left\|f \mid L^{p}(\Omega)\right\| & \leq\left\|\delta_{\lambda} \tilde{f}-\widetilde{f} \mid L^{p}(\Omega)\right\| \\
& \leq \int_{1}^{\lambda} \alpha^{-1}\left\|\delta_{\alpha} \widetilde{x \cdot \nabla f} \mid L^{p}(\Omega)\right\| d \alpha \\
& \leq \int_{1}^{\lambda} \alpha^{-1-n / p}\left\|x \cdot \nabla f \mid L^{p}(\Omega)\right\| d \alpha \\
& =\frac{p}{n}\left(1-\lambda^{-n / p}\right)\left\|x \cdot \nabla f \mid L^{p}(\Omega)\right\|,
\end{aligned}
$$

which implies (1.5) by letting $\lambda \rightarrow \infty$. 
Proof of Theorem 1.1. We first show (i). There exists some $\left\{f_{l}\right\}_{l=1}^{\infty} \subset C_{c}^{\infty}(\Omega)$ such that $f_{l} \rightarrow f$ in $W^{1, p}(\Omega)$ as $l \rightarrow \infty$. Let $\lambda>0$. We observe from Proposition 2.1 that

$$
\left\|\zeta_{\lambda} f_{l} \mid L^{p}(\Omega)\right\| \leq \frac{p}{n}\left\{\left\|\left(x \cdot \nabla \zeta_{\lambda}\right) f_{l}\left|L^{p}(\Omega)\|+\| \zeta_{\lambda}\left(x \cdot \nabla f_{l}\right)\right| L^{p}(\Omega)\right\|\right\}
$$

for all $l \geq 1$. We easily see that

$$
\begin{aligned}
\lim _{l \rightarrow \infty} \zeta_{\lambda} f_{l} & =\zeta_{\lambda} f \\
\lim _{l \rightarrow \infty}\left(x \cdot \nabla \zeta_{\lambda}\right) f_{l} & =\left(x \cdot \nabla \zeta_{\lambda}\right) f \\
\lim _{l \rightarrow \infty} \zeta_{\lambda}\left(x \cdot \nabla f_{l}\right) & =\lim _{l \rightarrow \infty}\left(\zeta_{\lambda} x\right) \cdot\left(\nabla f_{l}\right)=\zeta_{\lambda}(x \cdot \nabla f)
\end{aligned}
$$

in $L^{p}(\Omega)$. Hence we obtain

$$
\left\|\zeta_{\lambda} f \mid L^{p}(\Omega)\right\| \leq \frac{p}{n}\left\{\left\|\left(x \cdot \nabla \zeta_{\lambda}\right) f\left|L^{p}(\Omega)\|+\| \zeta_{\lambda}(x \cdot \nabla f)\right| L^{p}(\Omega)\right\|\right\} .
$$

Since

$$
\left|\left(x \cdot \nabla \zeta_{\lambda}\right)(x)\right| \leq\left\{\begin{array}{cc}
\|x \cdot \nabla \zeta\|_{\infty} & \text { if } \lambda^{-1}<|x|<2 \lambda^{-1} \\
0 & \text { otherwise }
\end{array}\right.
$$

(1.5) holds as $\lambda \rightarrow 0$.

We next prove (ii). We have only to prove that

$$
\begin{aligned}
\lim _{\lambda \rightarrow 0} \zeta_{\lambda}\left(f * \varphi_{\lambda}\right) & =f, \\
\lim _{\lambda \rightarrow 0} x \cdot \nabla\left(\zeta_{\lambda}\left(f * \varphi_{\lambda}\right)\right) & =x \cdot \nabla f
\end{aligned}
$$

in $L^{p}$ because $f_{\lambda} \equiv \zeta_{\lambda}\left(f * \varphi_{\lambda}\right) \in C_{c}^{\infty}\left(\mathbb{R}^{n}\right)$. We obviously have (2.4).

It follows that

$$
\begin{aligned}
x \cdot \nabla\left(\zeta_{\lambda}\left(f * \varphi_{\lambda}\right)\right) & =\left(x \cdot \nabla \zeta_{\lambda}\right)\left(f * \varphi_{\lambda}\right)+\zeta_{\lambda}(x \cdot \nabla)\left(f * \varphi_{\lambda}\right) \\
& =\left(x \cdot \nabla \zeta_{\lambda}\right)\left(f * \varphi_{\lambda}\right)+\zeta_{\lambda}\left((x \cdot \nabla f) * \varphi_{\lambda}\right)+\zeta_{\lambda}\left(f * \operatorname{div}\left(x \varphi_{\lambda}\right)\right) \\
& \equiv I_{\lambda}+I I_{\lambda}+I I I_{\lambda} .
\end{aligned}
$$

By (2.3), we have $\lim _{\lambda \rightarrow 0} I_{\lambda}=0$. Repeating the same argument of the proof of (2.4), we obtain $\lim _{\lambda \rightarrow 0} I I_{\lambda}=0$. Since $\int \operatorname{div}\left(x \varphi_{\lambda}\right)=0$, we see that

$$
\left(f * \operatorname{div}\left(x \varphi_{\lambda}\right)\right)(x)=\int_{\mathbb{R}^{n}}(f(x-\lambda y)-f(x) \operatorname{div}(y \varphi(y))) d y .
$$

Hence we obtain

$$
\left\|f * \operatorname{div}\left(x \varphi_{\lambda}\right)\right\|_{p} \leq \sup _{|z| \leq \lambda}\|f(\cdot-z)-f\|_{p}\|\operatorname{div}(x \varphi)\|_{1} \rightarrow 0
$$

as $\lambda \rightarrow 0$. Thus, we have $\lim _{\lambda \rightarrow 0} I I I_{\lambda}=0$. 
Finally, we show (iii). Since $f \in C^{1}\left(\mathbb{R}^{n}\right)$, we have

$$
f\left(e^{\theta} x\right)-f(x)=\int_{0}^{\theta} \frac{\partial}{\partial \alpha} f\left(e^{\alpha} x\right) d \alpha=\int_{0}^{\theta} e^{\alpha} x \cdot(\nabla f)\left(e^{\alpha} x\right) d \alpha
$$

for any $x \in \mathbb{R}^{n}$ and $\theta>0$. Let $\theta_{0}=\ln (R / \varepsilon)$ and let $|x| \geq \varepsilon$. Since $\left|e^{\theta_{0}} x\right| \geq R$, we see that $f\left(e^{\theta_{0}} x\right)=0$. By $(2.6)$, we have

$$
f(x)=-\int_{0}^{\theta_{0}} e^{\alpha} x \cdot(\nabla f)\left(e^{\alpha} x\right) d \alpha
$$

for any $|x| \geq \varepsilon$. Thus (1.7) holds.

\section{Application}

In this section, we show some corollaries of Proposition 1.3.

Corollary 3.1. Let $n \geq 1$ and $1 \leq p<\infty$. Assume that $f \in L_{\mathrm{loc}}^{p}\left(\mathbb{R}^{n}\right)$ satisfies $x \cdot \nabla f \in$ $L^{p}$. If there exist sequences $\left\{\rho_{l}\right\}_{l=1}^{\infty} \subset(0, \infty)$ and $\left\{R_{l}\right\}_{l=1}^{\infty} \subset(0, \infty)$ such that

(i) $R_{l} \leq R_{k}$ if $1 \leq l \leq k$,

(ii) $\lim _{l \rightarrow \infty} R_{l}=\infty$,

(iii)

$$
\liminf _{l \rightarrow \infty}\left(\frac{R_{l}+\rho_{l}}{\rho_{l}}\right)^{p} \int_{R_{l}<|x|<R_{l}+\rho_{l}}|f(x)|^{p} d x=0,
$$

then $f \in L^{p}$.

Remark 7. Taking $\rho_{l}=R_{l}$, we see from the above Corollary that if we have $x \cdot \nabla f \in L^{p}$ and

$$
\liminf _{R \rightarrow \infty} \int_{R<|x|<2 R}|f(x)|^{p} d x=0,
$$

then $f \in L^{p}$. If the left hand side of (3.2) is positive, then $f \notin L^{p}$ (even if $x \cdot \nabla f \in L^{p}$ ). Proof of Corollary 3.1. Set

$$
\phi_{l}(x)=\left\{\begin{array}{cc}
1 & \text { for }|x| \leq R_{l}, \\
\zeta\left(\frac{|x|-R_{l}+\rho_{l}}{\rho_{l}}\right) & \text { for }|x|>R_{l} .
\end{array}\right.
$$

We immediately see that

$$
\sup _{l \geq 1}\left\|\phi_{l}\right\|_{\infty}=1 \text { and } \lim _{l \rightarrow \infty} \phi_{l}(x)=1 \text { for a.e. } x \text {. }
$$


Since

$$
x \cdot \nabla \phi_{l}(x)=\left\{\begin{array}{cc}
\frac{|x|}{\rho_{l}} \zeta^{\prime}\left(\frac{|x|-R_{l}+\rho_{l}}{\rho_{l}}\right) & \text { if } R_{l}<|x|<R_{l}+\rho_{l}, \\
0 & \text { otherwise },
\end{array}\right.
$$

it follows that

$$
\liminf _{l \rightarrow \infty}\left\|\left(x \cdot \nabla \phi_{l}\right) f\right\|_{p}^{p} \leq \liminf _{l \rightarrow \infty}\left(\frac{R_{l}+\rho_{l}}{\rho_{l}}\right)^{p} \int_{R_{l}<|x|<R_{l}+\rho_{l}}|f(x)|^{p} d x=0 .
$$

Thus, we see from Proposition 1.3 that $f \in L^{p}$.

Corollary 3.2. Let $n \geq 1,1 \leq p<\infty$. Assume that $f \in L_{\text {loc }}^{p}\left(\mathbb{R}^{n}\right)$ satisfies $x \cdot \nabla f \in L^{p}$. If we have either of the following three conditions, then $f \in L^{p}$ :

(i) It follows that

$$
\lim _{R \rightarrow \infty} \sup _{R<|x|<2 R}|x|^{n / p}|f(x)|=0 .
$$

(ii) $p=1$ and there exists some spherically symmetric, unbounded open set $A$ such that

$$
\lim _{|x| \rightarrow \infty, x \in A}|x|^{n}|f(x)|=0 .
$$

(iii) $n=1, p=1$ and there exists some unbounded open set $A$ such that $-\inf A=$ $\sup A=\infty$ and (3.4) holds.

Remark 8. If

$$
\liminf _{|x| \rightarrow \infty}|x|^{n / p}|f(x)|>0,
$$

then $f \notin L^{p}$ even if $x \cdot \nabla f \in L^{p}$.

Proof of Corollary 3.2. We first prove (i). It follows that

$$
\begin{aligned}
\int_{R<|x|<2 R}|f(x)|^{p} d x & =\int_{R<|x|<2 R}\left(|x|^{n / p}|f(x)|\right)^{p}|x|^{-n} d x \\
& \leq\left(\sup _{R<|x|<2 R}|x|^{n / p}|f(x)|\right)^{p} \int_{R<|x|<2 R}|x|^{-n} d x \\
& \leq \ln 2\left(\sup _{R<|x|<2 R}|x|^{n / p}|f(x)|\right)^{p}\left(\int_{\mathbb{S}^{n-1}} d \omega\right) \\
& \rightarrow 0 \quad \text { as } R \rightarrow \infty .
\end{aligned}
$$

From Remark 7, we have $f \in L^{p}$.

We next show (ii). There exist $\left\{\rho_{l}\right\}_{l=1}^{\infty} \subset(0,1)$ and $\left\{R_{l}\right\}_{l=1}^{\infty} \subset(0, \infty)$ such that $\lim _{l \rightarrow \infty} \rho_{l}=0, R_{l} \leq R_{k}$ if $1 \leq l \leq k, \lim _{l \rightarrow \infty} R_{l}=\infty$ and

$$
A_{l} \equiv\left\{x \in \mathbb{R}^{n} ; R_{l}<|x|<R_{l}+\rho_{l}\right\} \subset A .
$$


Then we obtain

$$
\begin{aligned}
\frac{R_{l}+\rho_{l}}{\rho_{l}} & \int_{R_{l}<|x|<R_{l}+\rho_{l}}|f(x)| d x \\
& \leq \frac{R_{l}+\rho_{l}}{\rho_{l}}\left(\sup _{R_{l}<|x|<R_{l}+\rho_{l}}|x|^{n}|f(x)|\right)\left(\int_{\mathbb{S}^{n-1}} d \omega\right) \int_{R_{l}}^{R_{l}+\rho_{l}} r^{-1} d r \\
& \leq\left(\sup _{|x|>R_{l}, x \in A}|x|^{n}|f(x)|\right)\left(\int_{\mathbb{S}^{n-1}} d \omega\right) \frac{R_{l}+\rho_{l}}{\rho_{l}} \cdot \rho_{l} \cdot \frac{1}{R_{l}} \\
& \rightarrow 0 \quad \text { as } l \rightarrow \infty
\end{aligned}
$$

Applying Corollary 3.1, we see that $f \in L^{1}$.

Finally, we prove (iii). There exist $\left\{x_{l}\right\}_{l=1}^{\infty} \subset \mathbb{R}$ and $\left\{r_{l}\right\}_{l=1}^{\infty} \subset(0,1)$ such that

$$
\begin{aligned}
1<x_{2 k-1}<x_{2 k+1} & \text { for any } k \geq 1, \\
-1>x_{2 k}>x_{2 k+2} & \text { for any } k \geq 1, \\
\left|r_{l}\right| \rightarrow 0 & \text { as } l \rightarrow \infty \\
\left\{x \in \mathbb{R} ;\left|x-x_{l}\right|<r_{l}\right\} \subset A & \text { for any } l \geq 1 .
\end{aligned}
$$

Set

$$
\begin{aligned}
& \phi_{1}(x)=\left\{\begin{array}{cl}
\zeta(x) & \text { if } x<0 \\
1 & \text { if } 0 \leq x \leq x_{1} \\
\zeta\left(\frac{x-x_{1}+r_{1}}{r_{1}}\right) & \text { if } x>x_{1}
\end{array}\right. \\
& \phi_{2 k}(x)=\left\{\begin{array}{cl}
\zeta\left(\frac{x+x_{2 k}-r_{2 k}}{r_{2 k}}\right) & \text { if } x<x_{2 k}, \\
1 & \text { if } x_{2 k} \leq x \leq 0 \\
\phi_{2 k-1}(x) & \text { if } x>0
\end{array}\right. \\
& \phi_{2 k+1}(x)=\left\{\begin{array}{cl}
\phi_{2 k}(x) & \text { if } x<0, \\
1 & \text { if } 0 \leq x \leq x_{2 k+1}, \\
\zeta\left(\frac{x-x_{2 k+1}+r_{2 k+1}}{r_{2 k+1}}\right) & \text { if } x>x_{2 k+1},
\end{array}\right.
\end{aligned}
$$

where $k=1,2, \cdots$. Then we have

$$
\begin{aligned}
\sup _{l \geq 1}\left\|\phi_{l}\right\|_{\infty}=1, & \\
\phi_{l} f \in L^{p} & \text { for any } l \geq 1, \\
\lim _{l \rightarrow \infty} \phi_{l}(x)=1 & \text { for all } x \in \mathbb{R} .
\end{aligned}
$$


Furthermore, we see that

$$
x \cdot \nabla \phi_{2 k+1}=\left\{\begin{array}{cl}
0 & \text { if } x<x_{2 k}-r_{2 k}, \\
\frac{x}{r_{2 k}} \zeta^{\prime}\left(\frac{x+x_{2 k}-r_{2 k}}{r_{2 k}}\right) & \text { if } x_{2 k}-r_{2 k} \leq x \leq x_{2 k}, \\
0 & \text { if } x_{2 k}<x<x_{2 k+1}, \\
\frac{x}{r_{2 k+1}} \zeta^{\prime}\left(\frac{x-x_{2 k+1}+r_{2 k+1}}{r_{2 k+1}}\right) & \text { if } x_{2 k+1} \leq x \leq x_{2 k+1}+r_{2 k+1}, \\
0 & \text { if } x>x_{2 k+1}+r_{2 k+1},
\end{array}\right.
$$

for any $k \geq 1$. Thus, we obtain

$$
\begin{aligned}
\|(x & \left.\cdot \nabla \phi_{2 k+1}\right) f \|_{1} \\
\leq & \frac{-x_{2 k}+r_{2 k}}{r_{2 k}} \int_{x_{2 k}-r_{2 k}}^{x_{2 k}}|f(x)| d x+\frac{x_{2 k+1}+r_{2 k+1}}{r_{2 k+1}} \int_{x_{2 k+1}}^{x_{2 k+1}+r_{2 k+1}}|f(x)| d x \\
\leq & \frac{-x_{2 k}+r_{2 k}}{r_{2 k}} \cdot r_{2 k} \cdot \frac{1}{-x_{2 k}}\left(\sup _{x_{2 k}-r_{2 k} \leq x \leq x_{2 k}}|x \| f(x)| d x\right) \\
& +\frac{x_{2 k+1}+r_{2 k+1}}{r_{2 k+1}} \cdot r_{2 k+1} \cdot \frac{1}{x_{2 k+1}}\left(\sup _{x_{2 k+1} \leq x \leq x_{2 k+1}+r_{2 k+1}}|x||f(x)| d x\right) .
\end{aligned}
$$

Hence we observe that

$$
\liminf _{l \rightarrow \infty}\left\|\left(x \cdot \nabla \phi_{l}\right) f\right\|_{1}=0 .
$$

From Proposition 1.3, we have $f \in L^{1}$.

\section{REFERENCES}

[1] Adimurthi, N. Chaudhuri and M. Ramaswamy, An improved Hardy-Sobolev inequality and its application. Proc. Amer. Math. Soc. 130 (2002), 489-505.

[2] H. Brezis and M. Marcus, Hardy's inequalities revisited, Ann. Scuola Norm. Sup. Pisa Cl. Sci. (4) 25 (1997), 217-237.

[3] T. Cazenave, Semilinear Schrödinger Equations, American Mathematical Society, 2003.

[4] J. García Azorero and I. Peral Alonso, Hardy inequalities and some critical elliptic and parabolic problems, J. Differential Equations 144 (1998), 441-476.

[5] D. Gilbarg and N. S. Trudinger, Elliptic Partial Differential Equations of Second Order, Grundlehren 224, Springer-Verlag, Berlin, 1983.

[6] G. H. Hardy, Notes on a theorem of Hilbert, Math. Z. 6 (1920), 314-317.

[7] B. Muckenhoupt, Weighted norm inequalities for the Fourier transform, Trans. Amer. Math. Soc. 276 (1983), 729-742.

[8] F. Planchon, Sur un inégalité de type Poincaré, C. R. Acad. Sci. Paris Sr. I Math. 330 (2000), 21-23.

[9] J. Zhang, Extensions of Hardy inequality, J. Ineq. Appl. 2006 (2006), Article ID 69379, 5pages.

(T. Ozawa) Department of Mathematics, Hokkaido University, 060-0810, Japan. E-mail address: ozawa@math.sci.hokudai.ac.jp

(H.Sasaki) Department of Mathematics, Osaka University, 563-0043, Japan.

E-mail address: hisasaki@cr.math.sci.osaka-u.ac.jp 